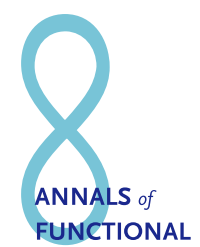

ANALYSIS
Ann. Funct. Anal. 7 (2016), no. 4, 678-685

http://dx.doi.org/10.1215/20088752-3661179

ISSN: 2008-8752 (electronic)

http://projecteuclid.org/afa

\title{
GATEAUX DERIVATIVE OF THE NORM IN $\mathcal{K}(X ; Y)$
}

\author{
PAWEŁ WÓJCIK
}

Communicated by G. Androulakis

\begin{abstract}
In this article, we consider the $\varphi$-Gateaux derivative of the norm in spaces of compact operators in such a way as to extend the Kečkić theorem. Our main result determines the $\varphi$-Gateaux derivative of the $\mathcal{K}(X ; Y)$ norm.
\end{abstract}

\section{INTRODUCTION AND PRELIMINARIES}

Let $(X,\|\cdot\|)$ be a normed space, and let $x, y \in X$. The directional derivative of the norm at $x$ in the $y$-direction is defined by

$$
D(x, y):=\lim _{t \rightarrow 0^{+}} \frac{\|x+t y\|-\|x\|}{t}, \quad x, y \in X .
$$

Convexity of the norm yields that the above definition is meaningful. The norm derivative is important in approximation theory and in the geometry of Banach spaces. In [6], the concept of $\varphi$-Gateaux derivatives was developed in order to substitute the usual concept of Gateaux derivatives at points which are not smooth. Let $\varphi \in[0,2 \pi)$, or let $\varphi \in\{0,-\pi\}$, if the space $X$ is over $\mathbb{R}$. The $\varphi$-Gateaux derivative of the norm at $x$ in the $\varphi, y$-direction is defined by

$$
D_{\varphi}(x, y):=\lim _{t \rightarrow 0^{+}} \frac{\left\|x+t e^{i \varphi} y\right\|-\|x\|}{t}, \quad x, y \in X .
$$

It is a straightforward verification to show that

$$
D_{\varphi}(x, y)=D\left(x, e^{i \varphi} y\right), \quad x, y \in X .
$$

Copyright 2016 by the Tusi Mathematical Research Group.

Received Mar. 31, 2016; Accepted Jul. 11, 2016.

2010 Mathematics Subject Classification. Primary 46B20; Secondary 47L05, 46 G05.

Keywords. space of compact operator, Gateaux derivative, dual space, adjoint operator, extreme point. 


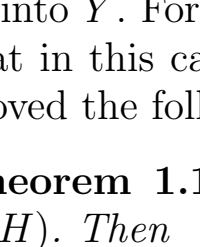

ANALYSIS
Ann. Funct. Anal. 7 (2016), no. 4, 678-685

http://dx.doi.org/10.1215/20088752-3661179

ISSN: 2008-8752 (electronic)

http://projecteuclid.org/afa

\title{
GATEAUX DERIVATIVE OF THE NORM IN $\mathcal{K}(X ; Y)$
}

\author{
PAWEŁ WÓJCIK
}

Communicated by G. Androulakis

\begin{abstract}
In this article, we consider the $\varphi$-Gateaux derivative of the norm in spaces of compact operators in such a way as to extend the Kečkić theorem. Our main result determines the $\varphi$-Gateaux derivative of the $\mathcal{K}(X ; Y)$ norm.
\end{abstract}

\section{INTRODUCTION AND PRELIMINARIES}

Let $(X,\|\cdot\|)$ be a normed space, and let $x, y \in X$. The directional derivative of the norm at $x$ in the $y$-direction is defined by

$$
D(x, y):=\lim _{t \rightarrow 0^{+}} \frac{\|x+t y\|-\|x\|}{t}, \quad x, y \in X .
$$

Convexity of the norm yields that the above definition is meaningful. The norm derivative is important in approximation theory and in the geometry of Banach spaces. In [6], the concept of $\varphi$-Gateaux derivatives was developed in order to substitute the usual concept of Gateaux derivatives at points which are not smooth. Let $\varphi \in[0,2 \pi)$, or let $\varphi \in\{0,-\pi\}$, if the space $X$ is over $\mathbb{R}$. The $\varphi$-Gateaux derivative of the norm at $x$ in the $\varphi, y$-direction is defined by

$$
D_{\varphi}(x, y):=\lim _{t \rightarrow 0^{+}} \frac{\left\|x+t e^{i \varphi} y\right\|-\|x\|}{t}, \quad x, y \in X .
$$

It is a straightforward verification to show that

$$
D_{\varphi}(x, y)=D\left(x, e^{i \varphi} y\right), \quad x, y \in X .
$$

Copyright 2016 by the Tusi Mathematical Research Group.

Received Mar. 31, 2016; Accepted Jul. 11, 2016.

2010 Mathematics Subject Classification. Primary 46B20; Secondary 47L05, 46 G05.

Keywords. space of compact operator, Gateaux derivative, dual space, adjoint operator, extreme point. 


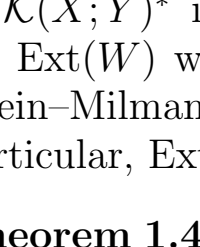

ANALYSIS
Ann. Funct. Anal. 7 (2016), no. 4, 678-685

http://dx.doi.org/10.1215/20088752-3661179

ISSN: 2008-8752 (electronic)

http://projecteuclid.org/afa

\title{
GATEAUX DERIVATIVE OF THE NORM IN $\mathcal{K}(X ; Y)$
}

\author{
PAWEŁ WÓJCIK
}

Communicated by G. Androulakis

\begin{abstract}
In this article, we consider the $\varphi$-Gateaux derivative of the norm in spaces of compact operators in such a way as to extend the Kečkić theorem. Our main result determines the $\varphi$-Gateaux derivative of the $\mathcal{K}(X ; Y)$ norm.
\end{abstract}

\section{INTRODUCTION AND PRELIMINARIES}

Let $(X,\|\cdot\|)$ be a normed space, and let $x, y \in X$. The directional derivative of the norm at $x$ in the $y$-direction is defined by

$$
D(x, y):=\lim _{t \rightarrow 0^{+}} \frac{\|x+t y\|-\|x\|}{t}, \quad x, y \in X .
$$

Convexity of the norm yields that the above definition is meaningful. The norm derivative is important in approximation theory and in the geometry of Banach spaces. In [6], the concept of $\varphi$-Gateaux derivatives was developed in order to substitute the usual concept of Gateaux derivatives at points which are not smooth. Let $\varphi \in[0,2 \pi)$, or let $\varphi \in\{0,-\pi\}$, if the space $X$ is over $\mathbb{R}$. The $\varphi$-Gateaux derivative of the norm at $x$ in the $\varphi, y$-direction is defined by

$$
D_{\varphi}(x, y):=\lim _{t \rightarrow 0^{+}} \frac{\left\|x+t e^{i \varphi} y\right\|-\|x\|}{t}, \quad x, y \in X .
$$

It is a straightforward verification to show that

$$
D_{\varphi}(x, y)=D\left(x, e^{i \varphi} y\right), \quad x, y \in X .
$$

Copyright 2016 by the Tusi Mathematical Research Group.

Received Mar. 31, 2016; Accepted Jul. 11, 2016.

2010 Mathematics Subject Classification. Primary 46B20; Secondary 47L05, 46 G05.

Keywords. space of compact operator, Gateaux derivative, dual space, adjoint operator, extreme point. 


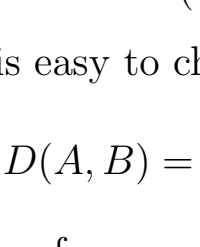

ANALYSIS
Ann. Funct. Anal. 7 (2016), no. 4, 678-685

http://dx.doi.org/10.1215/20088752-3661179

ISSN: 2008-8752 (electronic)

http://projecteuclid.org/afa

\title{
GATEAUX DERIVATIVE OF THE NORM IN $\mathcal{K}(X ; Y)$
}

\author{
PAWEŁ WÓJCIK
}

Communicated by G. Androulakis

\begin{abstract}
In this article, we consider the $\varphi$-Gateaux derivative of the norm in spaces of compact operators in such a way as to extend the Kečkić theorem. Our main result determines the $\varphi$-Gateaux derivative of the $\mathcal{K}(X ; Y)$ norm.
\end{abstract}

\section{INTRODUCTION AND PRELIMINARIES}

Let $(X,\|\cdot\|)$ be a normed space, and let $x, y \in X$. The directional derivative of the norm at $x$ in the $y$-direction is defined by

$$
D(x, y):=\lim _{t \rightarrow 0^{+}} \frac{\|x+t y\|-\|x\|}{t}, \quad x, y \in X .
$$

Convexity of the norm yields that the above definition is meaningful. The norm derivative is important in approximation theory and in the geometry of Banach spaces. In [6], the concept of $\varphi$-Gateaux derivatives was developed in order to substitute the usual concept of Gateaux derivatives at points which are not smooth. Let $\varphi \in[0,2 \pi)$, or let $\varphi \in\{0,-\pi\}$, if the space $X$ is over $\mathbb{R}$. The $\varphi$-Gateaux derivative of the norm at $x$ in the $\varphi, y$-direction is defined by

$$
D_{\varphi}(x, y):=\lim _{t \rightarrow 0^{+}} \frac{\left\|x+t e^{i \varphi} y\right\|-\|x\|}{t}, \quad x, y \in X .
$$

It is a straightforward verification to show that

$$
D_{\varphi}(x, y)=D\left(x, e^{i \varphi} y\right), \quad x, y \in X .
$$

Copyright 2016 by the Tusi Mathematical Research Group.

Received Mar. 31, 2016; Accepted Jul. 11, 2016.

2010 Mathematics Subject Classification. Primary 46B20; Secondary 47L05, 46 G05.

Keywords. space of compact operator, Gateaux derivative, dual space, adjoint operator, extreme point. 


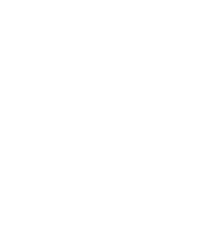

ANALYSIS
Ann. Funct. Anal. 7 (2016), no. 4, 678-685

http://dx.doi.org/10.1215/20088752-3661179

ISSN: 2008-8752 (electronic)

http://projecteuclid.org/afa

\title{
GATEAUX DERIVATIVE OF THE NORM IN $\mathcal{K}(X ; Y)$
}

\author{
PAWEŁ WÓJCIK
}

Communicated by G. Androulakis

\begin{abstract}
In this article, we consider the $\varphi$-Gateaux derivative of the norm in spaces of compact operators in such a way as to extend the Kečkić theorem. Our main result determines the $\varphi$-Gateaux derivative of the $\mathcal{K}(X ; Y)$ norm.
\end{abstract}

\section{INTRODUCTION AND PRELIMINARIES}

Let $(X,\|\cdot\|)$ be a normed space, and let $x, y \in X$. The directional derivative of the norm at $x$ in the $y$-direction is defined by

$$
D(x, y):=\lim _{t \rightarrow 0^{+}} \frac{\|x+t y\|-\|x\|}{t}, \quad x, y \in X .
$$

Convexity of the norm yields that the above definition is meaningful. The norm derivative is important in approximation theory and in the geometry of Banach spaces. In [6], the concept of $\varphi$-Gateaux derivatives was developed in order to substitute the usual concept of Gateaux derivatives at points which are not smooth. Let $\varphi \in[0,2 \pi)$, or let $\varphi \in\{0,-\pi\}$, if the space $X$ is over $\mathbb{R}$. The $\varphi$-Gateaux derivative of the norm at $x$ in the $\varphi, y$-direction is defined by

$$
D_{\varphi}(x, y):=\lim _{t \rightarrow 0^{+}} \frac{\left\|x+t e^{i \varphi} y\right\|-\|x\|}{t}, \quad x, y \in X .
$$

It is a straightforward verification to show that

$$
D_{\varphi}(x, y)=D\left(x, e^{i \varphi} y\right), \quad x, y \in X .
$$

Copyright 2016 by the Tusi Mathematical Research Group.

Received Mar. 31, 2016; Accepted Jul. 11, 2016.

2010 Mathematics Subject Classification. Primary 46B20; Secondary 47L05, 46 G05.

Keywords. space of compact operator, Gateaux derivative, dual space, adjoint operator, extreme point. 


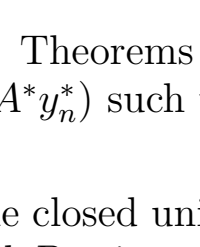

ANALYSIS
Ann. Funct. Anal. 7 (2016), no. 4, 678-685

http://dx.doi.org/10.1215/20088752-3661179

ISSN: 2008-8752 (electronic)

http://projecteuclid.org/afa

\title{
GATEAUX DERIVATIVE OF THE NORM IN $\mathcal{K}(X ; Y)$
}

\author{
PAWEŁ WÓJCIK
}

Communicated by G. Androulakis

\begin{abstract}
In this article, we consider the $\varphi$-Gateaux derivative of the norm in spaces of compact operators in such a way as to extend the Kečkić theorem. Our main result determines the $\varphi$-Gateaux derivative of the $\mathcal{K}(X ; Y)$ norm.
\end{abstract}

\section{INTRODUCTION AND PRELIMINARIES}

Let $(X,\|\cdot\|)$ be a normed space, and let $x, y \in X$. The directional derivative of the norm at $x$ in the $y$-direction is defined by

$$
D(x, y):=\lim _{t \rightarrow 0^{+}} \frac{\|x+t y\|-\|x\|}{t}, \quad x, y \in X .
$$

Convexity of the norm yields that the above definition is meaningful. The norm derivative is important in approximation theory and in the geometry of Banach spaces. In [6], the concept of $\varphi$-Gateaux derivatives was developed in order to substitute the usual concept of Gateaux derivatives at points which are not smooth. Let $\varphi \in[0,2 \pi)$, or let $\varphi \in\{0,-\pi\}$, if the space $X$ is over $\mathbb{R}$. The $\varphi$-Gateaux derivative of the norm at $x$ in the $\varphi, y$-direction is defined by

$$
D_{\varphi}(x, y):=\lim _{t \rightarrow 0^{+}} \frac{\left\|x+t e^{i \varphi} y\right\|-\|x\|}{t}, \quad x, y \in X .
$$

It is a straightforward verification to show that

$$
D_{\varphi}(x, y)=D\left(x, e^{i \varphi} y\right), \quad x, y \in X .
$$

Copyright 2016 by the Tusi Mathematical Research Group.

Received Mar. 31, 2016; Accepted Jul. 11, 2016.

2010 Mathematics Subject Classification. Primary 46B20; Secondary 47L05, 46 G05.

Keywords. space of compact operator, Gateaux derivative, dual space, adjoint operator, extreme point. 


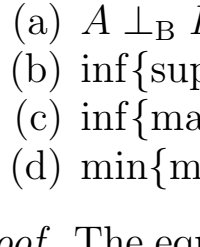

ANALYSIS
Ann. Funct. Anal. 7 (2016), no. 4, 678-685

http://dx.doi.org/10.1215/20088752-3661179

ISSN: 2008-8752 (electronic)

http://projecteuclid.org/afa

\title{
GATEAUX DERIVATIVE OF THE NORM IN $\mathcal{K}(X ; Y)$
}

\author{
PAWEŁ WÓJCIK
}

Communicated by G. Androulakis

\begin{abstract}
In this article, we consider the $\varphi$-Gateaux derivative of the norm in spaces of compact operators in such a way as to extend the Kečkić theorem. Our main result determines the $\varphi$-Gateaux derivative of the $\mathcal{K}(X ; Y)$ norm.
\end{abstract}

\section{INTRODUCTION AND PRELIMINARIES}

Let $(X,\|\cdot\|)$ be a normed space, and let $x, y \in X$. The directional derivative of the norm at $x$ in the $y$-direction is defined by

$$
D(x, y):=\lim _{t \rightarrow 0^{+}} \frac{\|x+t y\|-\|x\|}{t}, \quad x, y \in X .
$$

Convexity of the norm yields that the above definition is meaningful. The norm derivative is important in approximation theory and in the geometry of Banach spaces. In [6], the concept of $\varphi$-Gateaux derivatives was developed in order to substitute the usual concept of Gateaux derivatives at points which are not smooth. Let $\varphi \in[0,2 \pi)$, or let $\varphi \in\{0,-\pi\}$, if the space $X$ is over $\mathbb{R}$. The $\varphi$-Gateaux derivative of the norm at $x$ in the $\varphi, y$-direction is defined by

$$
D_{\varphi}(x, y):=\lim _{t \rightarrow 0^{+}} \frac{\left\|x+t e^{i \varphi} y\right\|-\|x\|}{t}, \quad x, y \in X .
$$

It is a straightforward verification to show that

$$
D_{\varphi}(x, y)=D\left(x, e^{i \varphi} y\right), \quad x, y \in X .
$$

Copyright 2016 by the Tusi Mathematical Research Group.

Received Mar. 31, 2016; Accepted Jul. 11, 2016.

2010 Mathematics Subject Classification. Primary 46B20; Secondary 47L05, 46 G05.

Keywords. space of compact operator, Gateaux derivative, dual space, adjoint operator, extreme point. 


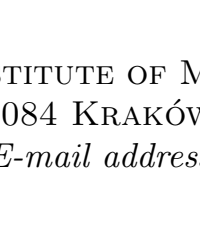

ANALYSIS
Ann. Funct. Anal. 7 (2016), no. 4, 678-685

http://dx.doi.org/10.1215/20088752-3661179

ISSN: 2008-8752 (electronic)

http://projecteuclid.org/afa

\title{
GATEAUX DERIVATIVE OF THE NORM IN $\mathcal{K}(X ; Y)$
}

\author{
PAWEŁ WÓJCIK
}

Communicated by G. Androulakis

\begin{abstract}
In this article, we consider the $\varphi$-Gateaux derivative of the norm in spaces of compact operators in such a way as to extend the Kečkić theorem. Our main result determines the $\varphi$-Gateaux derivative of the $\mathcal{K}(X ; Y)$ norm.
\end{abstract}

\section{INTRODUCTION AND PRELIMINARIES}

Let $(X,\|\cdot\|)$ be a normed space, and let $x, y \in X$. The directional derivative of the norm at $x$ in the $y$-direction is defined by

$$
D(x, y):=\lim _{t \rightarrow 0^{+}} \frac{\|x+t y\|-\|x\|}{t}, \quad x, y \in X .
$$

Convexity of the norm yields that the above definition is meaningful. The norm derivative is important in approximation theory and in the geometry of Banach spaces. In [6], the concept of $\varphi$-Gateaux derivatives was developed in order to substitute the usual concept of Gateaux derivatives at points which are not smooth. Let $\varphi \in[0,2 \pi)$, or let $\varphi \in\{0,-\pi\}$, if the space $X$ is over $\mathbb{R}$. The $\varphi$-Gateaux derivative of the norm at $x$ in the $\varphi, y$-direction is defined by

$$
D_{\varphi}(x, y):=\lim _{t \rightarrow 0^{+}} \frac{\left\|x+t e^{i \varphi} y\right\|-\|x\|}{t}, \quad x, y \in X .
$$

It is a straightforward verification to show that

$$
D_{\varphi}(x, y)=D\left(x, e^{i \varphi} y\right), \quad x, y \in X .
$$

Copyright 2016 by the Tusi Mathematical Research Group.

Received Mar. 31, 2016; Accepted Jul. 11, 2016.

2010 Mathematics Subject Classification. Primary 46B20; Secondary 47L05, 46 G05.

Keywords. space of compact operator, Gateaux derivative, dual space, adjoint operator, extreme point. 